\title{
Autoconfianza de los estudiantes de técnico en enfermería a partir de una experiencia clínica simulada
}

Camila P. Gatica-Videla1 ${ }^{1}$, Ivette N. Ilufi-Aguilera ${ }^{1}$ y Marta I. Fuentealba-Cruz ${ }^{\star}$

(1) Área de Salud. Universidad Santo Tomás. Talca-Chile. (correo-e: cgatica6@santotomas.cl; ivetteilufi@santotomas.cl)

(2) Facultad de Ciencias Básicas. Dpto. Biología y Química. Universidad Católica del Maule. Talca-Chile.

(correo-e: mfuentea@ucm.cl)

* Autor a quien debe ser dirigida la correspondencia

Recibido Abr. 5, 2021; Aceptado Jun. 7, 2021; Versión final Ago. 6, 2021, Publicado Oct. 2021

\section{Resumen}

Se evalúa el efecto de una secuencia didáctica que incorporó la utilización de simulación clínica de baja fidelidad con apoyo de tecnología, con diferentes escenarios virtuales que reunía diversos casos clínicos. Esto con la finalidad de analizar el impacto de esta herramienta pedagógica en el nivel de autoconfianza adquirido por estudiantes de la carrera de Técnico en Enfermería. Para ello se aplicó, antes y después de implementar la secuencia en forma remota virtual, la escala de autoconfianza (self-confidence scale), a 89 estudiantes que cursaban la asignatura Práctica de Enfermería II. Los resultados indican que después de la intervención, los estudiantes incrementaron su nivel de autoconfianza, presentándose el mayor aumento en la dimensión capacidad de intervención de un paciente (18.22\%), es decir, en la capacidad de ejecución de destrezas y habilidades motrices, competencia fundamental a desarrollar a nivel técnico. Se concluye que es aconsejable la incorporación de la simulación clínica de baja fidelidad de manera sistemática en el currículo de las carreras técnicas del área de salud.

Palabras clave: competencia; enseñanza-aprendizaje; secuencia didáctica; simulación clínica; autoconfianza

\section{Self-confidence of nursing technician students from a simulated clinical experience}

\begin{abstract}
In this article, we evaluate the effect of a didactic sequence, which incorporated the use of low-fidelity clinical simulation with the support of technology, with different virtual scenarios that gathered various clinical cases, the purpose of which was to analyze the impact of this pedagogical tool on the level of self-confidence acquired by students of the Nursing Technician career. For this, the self-confidence scale was applied, before and after implementing the sequence, to 89 students who were taking the Nursing Practice II course, in the second year of their degree. The results indicate that after the intervention, the students increase their level of selfconfidence, presenting the greatest increase in the dimension "intervention capacity" (18.22\%), that is, in the ability to execute motor skills and abilities, fundamental competence to develop at a technical level. It is concluded that it is necessary to systematically incorporate low-fidelity clinical simulation into the curriculum of technical careers in the health area.
\end{abstract}

Keywords: competence; teaching-learning; didactic sequence; clinical simulation; self-confidence 


\section{INTRODUCCIÓN}

La simulación clínica es una estrategia didáctica de amplia difusión y desarrollo que se emplea desde mediados de 1960, como un instrumento de formación en los estudiantes de Ciencias de la Salud (Serna y Martínez, 2018). Esta herramienta no reemplaza la práctica clínica habitual, sino que complementa, innova y enriquece los currículos de las carreras del área; brindando nuevas posibilidades de enseñanza - aprendizaje, permitiendo aumentar la seguridad del paciente y la autoconfianza en los estudiantes durante su formación académica; ya que requieren de escenarios en los que puedan consolidar los conocimientos, desarrollar competencias de modo seguro, sin causar daño al paciente (Allan et al., 2010; Coyer et al., 2014; Rubio et al., 2014) además, proporciona un entorno seguro para reorganizar el cuidado en salud y trabajar en equipo. Juguera et al. (2014) señalaron que la simulación clínica favorece la interacción de conocimientos, habilidades y factores humanos, con el fin de proporcionar un método de aprendizaje y entrenamiento efectivo, que logra desarrollar una práctica análoga a la que el estudiantado desarrollará en la realidad asistencial.

Concomitantemente, Martínez y Matus (2015) en una revisión bibliográfica sobre el desarrollo de habilidades con simulación clínica de alta fidelidad en estudiantes de enfermería, señalaron que esta estrategia permitió a los estudiantes adquirir experiencia del trabajo en equipo, asumir roles de liderazgo, asignar, delegar y acatar tareas, con el fin de atender a un paciente; además, indicaron que esta metodología vincula la teoría y la práctica, permitiendo la formación de equipos de trabajo y brindando seguridad ante situaciones de crisis. Asimismo, Ávila et al. (2020) afirma que es una estrategia didáctica y evaluativa que se ha desarrollado de manera exitosa, considerada con mucha relevancia en los currícula de las carreras profesionales del área de la salud, como un criterio de calidad educativa, ya que reduce la brecha existente entre la teoría y la práctica, fortaleciendo las competencias genéricas y permitiendo que el estudiante asuma responsabilidad en su formación. Por lo que el aprendizaje a través de simulación e interacción con elementos clínicos en ambientes simulados, se ha convertido en una herramienta pedagógica de alta efectividad en el campo clínico, para la preparación de profesionales competentes, capaces de mejorar los procesos de atención e intervención (Weller, 2012). La literatura describe diferentes modelos educativos que se utilizan en simulación clínica y por ejemplo: 1) simuladores en forma de fantoma, que proporcionan elementos de retroalimentación; 2) simuladores virtuales y programas de computación, que son utilizados para aumentar las habilidades prácticas de los estudiantes en procesos de intervención invasiva en pacientes y 3) entornos controlados con aparatos para la medición de habilidades clínicas, herramienta ampliamente utilizada en la educación basada en casos clínicos (Kawarai et al., 2020).

Por otra parte, es importante mencionar que existen diferentes niveles de fidelidad en la simulación clínica, el cual define el grado de realismo de los modelos y de la experiencia en la que se usan, entre ellas están: la de baja, mediana y alta fidelidad (Ávila et al., 2020). Corvetto et al. (2013) señaló que la de baja fidelidad simula solo una parte del organismo y se utiliza para el desarrollo de habilidades técnicas de un procedimiento, por ejemplo, la instalación de una vía venosa periférica. La de mediana fidelidad combina el uso de una parte anatómica con programas computacionales, que permiten al instructor manejar variables fisiológicas con el fin de desarrollar una competencia, por ejemplo, la reanimación cardiopulmonar. Y la de alta fidelidad integra múltiples variables fisiológicas para crear escenarios realistas con fantomas de tamaño real con el fin de desarrollar competencias técnicas, no técnicas y el manejo de crisis (Kawarai et al., 2020).

El avance de las nuevas tecnologías ha estimulado la creación de centros de simulación clínica en el ámbito de las ciencias de la salud, generando un beneficio tanto para los estudiantes, como para los pacientes, ya que la posibilidad de error se minimiza (Kononowicz et al., 2019). Actualmente, la gran mayoría de las carreras del área de la salud utilizan la simulación clínica como estrategia de enseñanza-aprendizaje, con el propósito de fortalecer el conocimiento, habilidades y competencias de sus estudiantes (Dearmon et al., 2013). Herramienta pedagógica que es ampliamente utilizada en la formación profesional, no así en la formación técnica del área de la salud. En Chile, a mediados de los años 90 se conocen los primeros usos de esta metodología, que permitía a los estudiantes del área de salud practicar procedimientos clínicos (Corvetto et al., 2013). Por otra parte, la creación de la Asociación Latinoamericana de Simulación Clínica (ALASIC) en el año 2007 y de la fundación de la reciente Sociedad Chilena De Simulación Clínica y Seguridad del Paciente (SOCHISIM) son hitos que demuestran el desarrollo de esta estrategia de enseñanza en la última década en Latinoamérica y especialmente en Chile (Martínez y Matus, 2015).

Si bien, la literatura muestra un gran interés por el estudio sobre el nivel de autoconfianza, entendiéndola como el juicio o autopercepción sobre la propia capacidad (Haro, 2019), que adquieren los estudiantes sometidos a simulación clínica (Cheng et al., 2015; Kawarai et al., 2020), herramienta didáctica que ha sido implementada en áreas como anestesiología, ortopedia, fisioterapia, terapia ocupacional, terapia respiratoria, fonoaudiología, neonatología, obstetricia, entre otras (Huang et al., 2019; Mundell et al., 2013), no hemos encontrado reportes en que se utilice esta herramienta pedagógica-didáctica en la formación de técnicos en enfermería, incluso (y especialmente) la simulación clínica de baja fidelidad que utiliza modelos que simulan sólo una parte del organismo, usados generalmente para adquirir habilidades motrices básicas en un 
procedimiento simple o examen físico (Corvetto et al., 2013; House et al., 2016). En este escenario emerge de manera natural la necesidad de diseñar e incorporar estrategias de enseñanza, que incorporen la simulación clínica de baja fidelidad en la formación de técnicos en enfermería de nivel superior (TENS), ya que son actores vitales en la estructura de la organización de los centros de salud.

En este sentido, este artículo presenta un estudio en el que se diseñó y aplicó una secuencia didáctica basada en simulación clínica de baja fidelidad con apoyo de tecnología, a estudiantes de educación superior (técnico profesional) de la carrera Técnico en Enfermería, con el propósito de fomentar la autoconfianza en las distintas dimensiones de su quehacer laboral. Entre las razones que justifican la elección de la intervención, es que las actividades que desarrollan los TENS son fundamentales en la interacción con los pacientes, además en el cumplimiento de los protocolos y normas sanitarias en general, y, en consecuencia, los futuros TENS se convierten en actores clave al momento de la prestación de cuidados de enfermería a los pacientes, a la familia y a la comunidad, en los diversos niveles de atención de la salud, con un enfoque biopsicosocial, ejecutando procedimientos de enfermería y de colaboración con el equipo de salud. De acuerdo con lo anterior, el objetivo del presente trabajo, es identificar y comparar la autoconfianza en el aprendizaje de los estudiantes de la carrera TENS, a partir de la simulación clínica de baja fidelidad como método de enseñanzaaprendizaje.

\section{METODOLOGÍA}

Se realizó un estudio cuasi-experimental, longitudinal en grupos intactos y todos los participantes componen el mismo curso. Se utilizó una secuencia didáctica que fue evaluada con un pre-test y un post-test.

\section{Muestra}

La muestra estuvo constituida por estudiantes de la carrera TENS del CFT Santo Tomás, sede Talca, modalidad diurna, que cursaban el segundo año de carrera, que tuvieran aprobadas las asignaturas (i) Práctica de Enfermería Básica I, (ii) Enfermería Médico Quirúrgica y (iii) Administración de Fármacos y que estuvieran inscritos en la asignatura Práctica de Enfermería II, durante el primer semestre académico del año 2020. La inscripción fue de un total de 115 alumnos para dicha actividad curricular. Los participantes fueron seleccionados mediante un muestreo probabilístico aleatorio simple, con aplicación de random. Para la selección del tamaño de la muestra se utilizó el programa Decision Analyst STATS 2.0, obteniendo un tamaño de muestra de 89 estudiantes con un nivel de confianza de $95 \%$ y error máximo de 5\%, cuyas edades oscilan entre 18 a 20 años, siendo el $80,9 \%$ mujeres y el $19 \%$ varones.

\section{Consideraciones éticas}

La realización de este estudio fue autorizada por la Dirección de Postgrado, Dirección Nacional de Simulación Clínica y Seguridad del Paciente del CFT Santo Tomás y por la Dirección de Carrera de la sede involucrada. Todos los participantes firmaron de manera voluntaria un consentimiento informado, garante de resguardar sus datos personales, pudiendo solicitar ser excluido y que sus intervenciones no fueran consideradas en la investigación sin justificación previa, ni perjuicio para el participante. Cabe señalar que se tomaron todas las medidas para garantizar la salud, integridad y bienestar de los participantes_.

\section{Instrumento}

Se utilizó la escala Self - Confidence Scale (SCS) de Hicks et al. (2009) previa autorización del autor. La traducción del inglés al español de la SCS la realizaron profesionales bilingües para adaptarla a nuestro contexto. La SCS consta de 12 preguntas, cuyas respuestas se miden solicitando el grado de acuerdo para cada ítem en formato tipo Likert de 5 puntos: (1) nada seguro, (2) poco seguro, (3) algo seguro, (4) moderadamente seguro y (5) muy seguro. Los ítems están organizados en 4 dimensiones: 1) Reconocimiento de signos y síntomas: implica reconocer con precisión un cambio en las condiciones de los pacientes con problemas cardíacos, respiratorios y neurológicos; 2) Valoración de la condición del paciente: implica realización de evaluaciones físicas básicas de la condición de los pacientes con problemas cardíacos, respiratorios y neurológicos; 3) Capacidad de intervención: implica ejecutar o realizar cuidados de enfermería en el paciente a través de la identificación de intervenciones básicas de enfermería en los pacientes con problemas cardíacos, respiratorios y neurológicos; y 4) Evaluación del paciente: involucra la evaluación de la eficacia de las intervenciones ejecutadas para las condiciones de los pacientes con problemas cardíacos, respiratorios y neurológicos.

\section{Secuencia didáctica}

La secuencia didáctica (Tabla 1), se realizó con un total de 88 horas pedagógicas y constó de diferentes etapas que contienen actividades diversas con propósitos particulares, estas son: 
Etapa I: Se realizó una charla introductoria para presentar las etapas que conforman la secuencia didáctica, que son el pre-debriefing, simulación clínica y debriefing. Se socializó las guías talleres que los estudiantes debían revisar previo a la simulación clínica de baja fidelidad virtual. Se discutió el método de evaluación y se formaron los equipos de trabajo. Durante el pre-debriefing se dieron a conocer los objetivos, los resultados de aprendizaje esperados, las competencias y habilidades a desarrollar en cada etapa de la secuencia didáctica. Por otra parte, se realizó una lluvia de ideas en relación con las temáticas a desarrollar, por ejemplo, materiales de la técnica, etapas del procedimiento, cuidados de enfermería, rol del técnico, entre otros. Por último, se contextualizó el escenario virtual (simulación clínica) mediante un contrato ficticio. El objetivo de esta etapa fue aunar los conocimientos de los estudiantes, entregando las bases para contextualizar la metodología. La duración de esta etapa fue de 4 horas pedagógicas.

Etapa II: Los estudiantes visualizaron el escenario remoto virtual, cuya temática reunía casos clínicos de enfermería médico-quirúrgica. La duración de esta etapa fue de 36 horas pedagógicas. Al finalizar cada escenario, los estudiantes se reunieron con su equipo de trabajo y analizaron el caso clínico presentado, considerando las decisiones, valoración y atención de enfermería brindada por el técnico en enfermería. Además, se discutió las técnicas realizadas en el caso clínico, realizando la discriminación de errores cometidos durante la atención al paciente. Por otra parte, cada estudiante realizó la autoevaluación del procedimiento, con el objeto de ser una instancia de mejora. Finalmente, el docente realizó la retroalimentación del taller y lo evaluó mediante una pauta de cotejo.

Etapa III: Los estudiantes visualizaron el escenario remoto virtual, cuya temática reunía casos clínicos de gineco-obstetricia. La duración de esta etapa fue de 28 horas pedagógicas. Al finalizar el escenario, se siguió el protocolo descrito en la etapa II.

Etapa IV: Los estudiantes visualizaron el escenario remoto virtual, cuya temática reunía casos clínicos de atención primaria. La duración de esta etapa fue de 20 horas pedagógicas. Al finalizar el escenario, se siguió el protocolo descrito en la etapa II.

Tabla 1. Actividades de la secuencia didáctica

\begin{tabular}{|c|c|c|c|c|c|}
\hline Etapa & $\begin{array}{l}\text { Objetivos de } \\
\text { aprendizaje }\end{array}$ & $\begin{array}{l}\text { Tiempo } \\
\text { (Horas) }\end{array}$ & Recursos & Escenarios Virtuales & Evaluación \\
\hline I & $\begin{array}{l}\text { Conocer la metodología } \\
\text { de simulación clínica. }\end{array}$ & 4 & $\begin{array}{l}\text { Docente facilitador } \\
\text { MS Teams }\end{array}$ & No aplica & No aplica \\
\hline II & $\begin{array}{l}\text { Planificar la atención de } \\
\text { enfermería médico- } \\
\text { quirúrgica integral. } \\
\text { Aplicar procedimientos } \\
\text { de enfermería básica. } \\
\text { Aplicar procedimientos } \\
\text { de enfermería médico- } \\
\text { quirúrgica. } \\
\text { Aplicar de medidas de } \\
\text { prevención de } \\
\text { infecciones. }\end{array}$ & 36 & $\begin{array}{l}\text { Docente facilitador } \\
\text { Guía-taller } \\
\text { Cápsulas educativas } \\
\text { MS Teams }\end{array}$ & $\begin{array}{l}\text { Atención de enfermería a } \\
\text { pacientes: } \\
\text { Con ataque } \\
\text { cerebrovascular. } \\
\text { Con neumonía. } \\
\text { Con insuficiencia renal. } \\
\text { Con diabetes mellitus tipo II. } \\
\text { Con infarto agudo al } \\
\text { miocardio. } \\
\text { Con intoxicación. } \\
\text { En preoperatorio inmediato. } \\
\text { En proceso postoperatorio. }\end{array}$ & $\begin{array}{l}\text { Autoevaluación } \\
\text { Evaluación de } \\
\text { pares } \\
\text { Pauta de cotejo }\end{array}$ \\
\hline III & $\begin{array}{l}\text { Aplicar procedimientos } \\
\text { en preparto, parto, } \\
\text { puerperio y ginecología. } \\
\text { Aplicar procedimientos } \\
\text { de enfermería básica. } \\
\text { Aplicar de medidas de } \\
\text { prevención de } \\
\text { infecciones. }\end{array}$ & 28 & $\begin{array}{l}\text { Docente facilitador } \\
\text { Guía-taller } \\
\text { Cápsulas educativas } \\
\text { MS Teams }\end{array}$ & $\begin{array}{l}\text { Atención de enfermería a } \\
\text { pacientes: } \\
\text { Con hemorragia post-parto. } \\
\text { Con síndrome hipertensivo } \\
\text { del embarazo. } \\
\text { Con diabetes gestacional. } \\
\text { Con diagnóstico de rotura } \\
\text { prematura de membranas. } \\
\text { En el ingreso de } \\
\text { embarazada a emergencia. }\end{array}$ & $\begin{array}{l}\text { Autoevaluación } \\
\text { Evaluación de } \\
\text { pares } \\
\text { Pauta de cotejo }\end{array}$ \\
\hline IV & $\begin{array}{l}\text { Aplicar procedimientos } \\
\text { de enfermería en } \\
\text { atención primaria. } \\
\text { Educar al paciente y } \\
\text { familiares. } \\
\text { Aplicar de medidas de } \\
\text { prevención de } \\
\text { infecciones asociadas a } \\
\text { la atención en salud. }\end{array}$ & 20 & $\begin{array}{l}\text { Docente facilitador } \\
\text { Guía-taller } \\
\text { Cápsulas educativas } \\
\text { MS Teams }\end{array}$ & $\begin{array}{l}\text { Recepción de paciente para } \\
\text { control cardiovascular. } \\
\text { Atención a adulto mayor con } \\
\text { Alzheimer. } \\
\text { Atención paciente en } \\
\text { ELEAM. } \\
\text { Atención paciente postrado } \\
\text { con diagnóstico de } \\
\text { demencia senil. }\end{array}$ & $\begin{array}{l}\text { Autoevaluación } \\
\text { Evaluación de } \\
\text { pares } \\
\text { Pauta de cotejo }\end{array}$ \\
\hline
\end{tabular}




\section{RESULTADOS}

Los datos obtenidos a partir de los instrumentos aplicados se analizaron con el software estadístico SPSS, versión 23 de IBM Statistics®.

\section{Fiabilidad del instrumento}

La fiabilidad original de la SCS basado en el coeficiente de alfa de Cronbach, obtenido desde el estudio de Hicks et al. (2009), fue de 0,93 para el pre-test y 0,96 para el post-test. En este estudio se obtuvo una fiabilidad de 0,847 para el pre-test y 0,837 para el post-test, lo que demuestra alta consistencia interna del instrumento, por lo que es altamente fiable.

\section{Escala de autoconfianza}

Al promediar los valores que entregaron los estudiantes a las diferentes dimensiones de la escala de autoconfianza, en el pre-test se detectó que la mayor valoración corresponde a la dimensión reconocimiento de signos y síntomas $(67.50 \%)$ y la menor, a la evaluación del paciente $(56.48 \%)$; tendencia que se mantiene en el post-test detectando que la mayor valoración corresponde a la dimensión reconocimiento de signos y síntomas (81.80\%) y la menor a la evaluación del paciente (74.40\%) (Tabla 2). De acuerdo con la valoración a través de la escala Likert, los valores de autoconfianza de los estudiantes obtenidos antes de aplicar la secuencia didáctica son bajos, variando de algo seguro (3.37 y 3.32) a poco seguro (2.96 y 2.82). Por su parte, la valoración de la autoconfianza después de aplicada la secuencia didáctica aumenta de moderadamente seguro (4.09 y 4.03 ) a algo seguro (3.87 y 3.72 ); las diferencias detectadas entre las distintas dimensiones son significativas $(p>0.001)$ (Tabla 2$)$.

Además, es importante observar que existe una tendencia inversa entre el aumento de la complejidad de la dimensión y la disminución de la autoconfianza, ya que, en el reconocimiento de signos y síntomas de un evento cardiaco, respiratorio y neurológico en un paciente, el promedio de autoconfianza es de $67.50 \%$ y $81.80 \%$ (pre y post test) y en la dimensión de evaluación del paciente, que valora la eficacia de sus intervenciones en usuarios con problemas cardiacos, respiratorios y neurológicos, el promedio de autoconfianza es de un $56.48 \%$ y $74.40 \%$ (pre y post test) (Tabla 2 ).

Tabla 2: Distribución numérica y porcentual de la población en estudio según dimensión de la escala de autoconfianza y su valoración a través de la escala de Likert.

\begin{tabular}{|l|c|c|c|c|}
\hline \multirow{2}{*}{ Dimensión } & \multicolumn{3}{|c|}{ Valoración de Likert } \\
\cline { 2 - 5 } & \multicolumn{2}{|c|}{ Pre-test } & \multicolumn{2}{c|}{ Post-test } \\
\cline { 2 - 5 } & $\begin{array}{c}\text { Valores escala } \\
\text { de Likert }\end{array}$ & Porcentaje & $\begin{array}{c}\text { Valores escala } \\
\text { de Likert }\end{array}$ & Porcentaje \\
\hline Reconocimiento de signos y síntomas & $3.37 \pm 0.89$ & 67.50 & $4.09 \pm 0.70$ & 81.80 \\
\hline Valoración de la condición del paciente & $3.20 \pm 0.87$ & 64.04 & $4.03 \pm 0.73$ & 80.60 \\
\hline Capacidad de intervención & $2.96 \pm 0.91$ & 59.18 & $3.87 \pm 0.82$ & 77.40 \\
\hline Evaluación del paciente & $2.82 \pm 0.87$ & 56.48 & $3.72 \pm 1.05$ & 74.40 \\
\hline
\end{tabular}

\section{Comparación entre el Pre y Post test}

Para realizar la comparación del nivel de autoconfianza antes y después de la intervención didáctica, previamente se determinó que las muestras no se distribuyen de manera normal según el test de normalidad de Kolmogorov-Smirnov $(p<0.05)$, por lo tanto, se utilizó la prueba de rangos con signos de Wilcoxon para muestras relacionadas, donde obtuvimos un valor $p<0.001$, por lo que pudimos detectar que existe una diferencia significativa entre los resultados del pre y post - test. Asimismo, sobre la base de la figura 1, podemos indicar que la intervención contribuyó a incrementar el nivel de autoconfianza de los estudiantes en cada una de las dimensiones de la escala, obteniendo una diferencia en el aumento del nivel de autoconfianza de entre un $14.30 \%$ en el reconocimiento de signos y síntomas y de un $18.22 \%$ en la capacidad de intervención de un paciente (Tabla 3). 


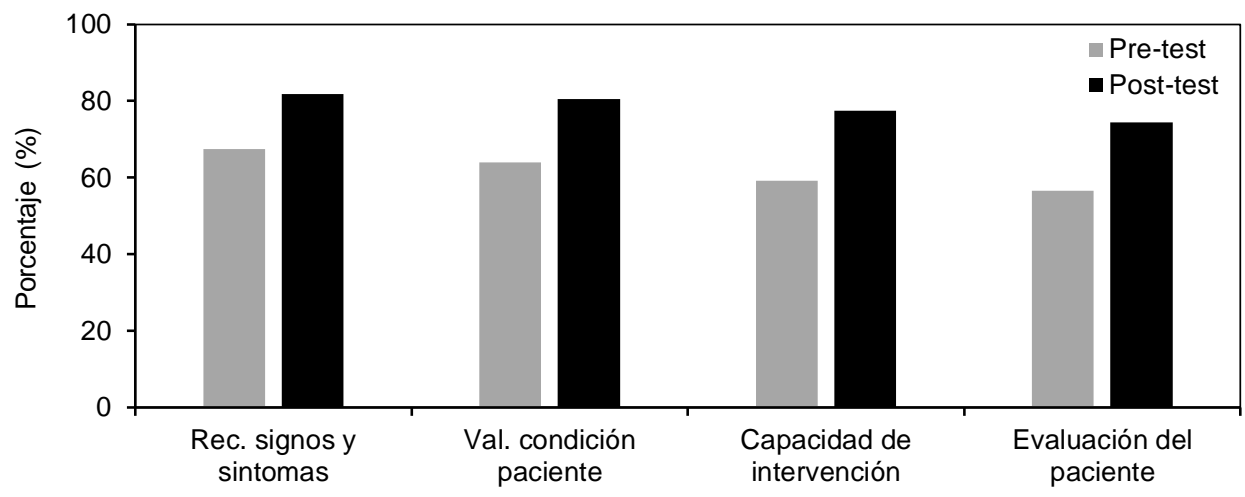

Dimensiones de la Escala de Autoconfianza

Fig. 1: Distribución porcentual de la valoración de las dimensiones de la escala de autoconfianza antes y después de la intervención didáctica.

Tabla 3: Comparación en porcentaje de las dimensiones de la escala de autoconfianza y su valoración, antes y después de la intervención.

\begin{tabular}{|c|c|c|c|}
\hline \multirow{2}{*}{ Dimensión } & \multicolumn{2}{|c|}{ Valoración de Likert (\%) } & \multirow{2}{*}{ Diferencia (\%) } \\
\hline & Pre-test & Post-test & \\
\hline Reconocimiento de signos y síntomas & 67.50 & 81.80 & 14.30 \\
\hline Valoración de la condición del paciente & 64.04 & 80.60 & 16.56 \\
\hline Capacidad de intervención & 59.18 & 77.40 & 18.22 \\
\hline Evaluación del paciente & 56.48 & 74.40 & 17.92 \\
\hline & & omedio total & 16.75 \\
\hline
\end{tabular}

\section{DISCUSIÓN}

Los escenarios de simulación clínica han sido ampliamente explorados en medicina y enfermería como estrategia de aprendizaje y han demostrado ser efectivos para la adquisición de competencias, acercando a los estudiantes a su práctica real (Afonso et al., 2020; Serna y Martínez, 2018) brindando nuevas posibilidades de enseñanza - aprendizaje, favoreciendo que los estudiantes adquieran conocimientos significativos (Kennedy et al., 2014; Brady et al., 2015) donde éste, es un actor partícipe del proceso de atención clínica en un ambiente determinado, controlado por el docente (Laschinger et al., 2008), permitiendo recrear escenarios lo más cercano a la realidad hospitalaria, sin exponer a los estudiantes a riesgos comunes, como por ejemplo enfermedades infecto-contagiosas. Por otra parte, se puede practicar un sin número de veces en los diversos simuladores, mejorar las técnicas sin lastimar o poner en riesgo a un paciente (Mould et al., 2011; House et al., 2016) fomentando la seguridad de los pacientes, como la autoconfianza en los estudiantes, ya que, este puede tomar decisiones, crear estrategias y resolver problemas de forma más eficiente en el quehacer de la profesión (Kononowicz et al., 2019). Por otro lado, Kawarai et al. (2020) en una revisión bibliográfica de 17 estudios sobre el efecto de la simulación de baja y de alta fidelidad para procedimientos no laparoscópicos, en las habilidades de procedimientos, detectaron que 15 de los 17 estudios mostraron que la simulación de baja fidelidad produjo resultados similares a la simulación de alta fidelidad; además concluyeron que los simuladores de baja fidelidad pueden proporcionar importantes beneficios educativos a estudiantes menos experimentados.

Los resultados obtenidos en el presente estudio nos indican que la intervención contribuyó a incrementar el nivel de autoconfianza de los estudiantes, en cada una de las dimensiones que mide la escala de Hick et al. (2009), detectando que el mayor aumento correspondió a la dimensión "capacidad de intervención de un paciente", es decir, la capacidad de ejecución de destrezas motrices, la cual es una competencia de nivel técnico, que está directamente relacionada con el perfil de egreso de la carrera de Técnico en Enfermería del CFT Santo Tomás. Por consiguiente, estos resultados nos permiten recomendar la incorporación de la simulación clínica de baja fidelidad, como herramienta pedagógica didáctica en el currículo de TENS, para potenciar mayor acercamiento de los estudiantes a los pacientes, con un entrenamiento inicial en escenarios simulados; con la finalidad de fomentar la autoconfianza y con ello destrezas y habilidades motrices en los estudiantes, ya que esta herramienta es una excelente estrategia didáctica para el aprendizaje de muchos de los contenidos conceptuales, procedimentales y actitudinales, incluyendo habilidades intelectuales, habilidades sociales, habilidades motrices, destrezas manipulativas y capacidades holísticas, entre otras. 
Considerando, además, que la práctica simulada de baja fidelidad promueve el principio de "no maleficiencia", i.e., no producir daño y prevenirlo (Weller, 2012), se hace necesario incorporarla sistemáticamente como una práctica deliberada con actividades acorde con el nivel curricular del estudiante, independiente de la disponibilidad y equipamiento del centro educacional. Al respecto, House et al. (2016) en un estudio realizado con estudiantes de enfermería, donde se evaluó una estrategia de simulación de baja fidelidad, señalaron la relevancia del análisis de los planes de estudio de las carreras de enfermería, considerando actividades que incorporen este método, con la finalidad de reforzar la seguridad del paciente. Por su parte, Brady et al. (2015) señalaron que la simulación, ya sea de alta o baja fidelidad, como enfoque pedagógico, promueve el desarrollo de manera segura habilidades clínicas efectivas antes de emprender la práctica clínica, en entornos de atención compleja.

Concomitantemente, es muy importante la elaboración de protocolos para el desarrollo de los talleres de simulación, que incorporen la metodología (pre-debriefing, escenario y debriefing) de acuerdo a la actividad académica a realizar; debido a que ayuda a los estudiantes a construir su propio aprendizaje como un proceso y a extrapolar lo aprendido a situaciones más generalistas mediante la reflexión, el análisis, la internalización y la relación que se establece en la etapa del debriefing (Zhang et al., 2020), así como la capacitación a los docentes, con el fin de ampliar sus conocimientos en la simulación y en la importancia del uso de estos documentos para optimizar dichos talleres (Ñáñez et al., 2019; Verkuyl et al., 2020).

Por otra parte, es importante comentar que los resultados del presente estudio han sido obtenidos en un escenario de salud pública incierto producto del COVID-19; lo que obligó a tomar medidas preventivas ajenas a nuestra cotidianeidad, como es la distancia física, lo que nos llevó a implementar la enseñanza telemática en su forma sincrónica como asincrónica. Lo cual ha sido una oportunidad para eliminar barreras espacio temporal en el proceso educativo, permitiendo que los estudiantes incrementen su autoconfianza aprovechando los elementos y recursos que ofrece esta tecnología; coincidiendo con un estudio realizado en la Universidad de Cundinamarca en Colombia, donde se examinaron el uso de las aulas virtuales en el campo educativo, allí docentes y estudiantes consideraron que es un espacio importante y útil en el proceso de enseñanza y aprendizaje (Martínez y Jiménez, 2020).

Cabe destacar, que se identificaron varias limitaciones en el estudio, entre ellas (i) la muestra de conveniencia de un Centro de Formación Técnica limita la capacidad de generalizar los resultados a otras poblaciones, (ii) falta de investigación en el área de simulación de baja fidelidad en la formación de TENS, por lo que los resultados no se pudieron comparar con investigaciones anteriores. Finalmente, podemos señalar que a la luz de los resultados se evidencia la apertura un campo de formación que garantiza mayor autoconfianza para la práctica clínica de los TENS, teniendo en cuenta que es necesario desarrollar trabajos en escenarios de simulación que favorezcan la adquisición y el fortalecimiento de competencias que promuevan el desempeño técnico-profesional, implementando simulación clínica de baja fidelidad, aportando el realismo necesario que permita trabajar la autoconfianza. De esta forma, se podrá mejorar el desempeño del TENS en su formación y su impacto en los diversos contextos clínicos y niveles de atención de salud.

\section{CONCLUSIONES}

La secuencia didáctica que incorporó la utilización de simulación clínica de baja fidelidad buscó concretar situaciones reales en contextos de enseñanza y contribuyó a incrementar el nivel de autoconfianza de los estudiantes de la carrera Técnico en Enfermería del CFT Santo Tomás en un 16.75\%, siendo la capacidad de intervención, la dimensión que mayormente se incrementó (18.22\%).

\section{REFERENCIAS}

Allan, C.K., Thiagarajan, R.R., y otros ocho autores, Simulation-based training delivered directly to the pediatric cardiac intensive care unit engenders preparedness, comfort, and decreased anxiety among multidisciplinary resuscitation teams, https://doi.org/10.1016/j.jtcvs.2010.04.027, J. Thorac. Cardiovasc. Surg., 140(3), 646-52 (2010)

Alfonso, M.L., Castellanos, G.L., y otros seis autores, Aprendizaje basado en simulación: estrategia pedagógica en fisioterapia. Revisión integrativa, https://doi.org/10.1016/J.EDUMED.2018.11.001, Educación Médica, 21(6), 357-363 (2020)

Ávila, S.A., García, A.M., y Morales, S., Simulación con paciente estandarizado y simuladores de baja fidelidad (PESiBaF) como primer acercamiento a un paciente en estudiantes de primer año de la carrera de médico cirujano, https://doi.org/10.1016/j.edumed.2018.10.011, Educación Médica, 21(6), 364-369 (2020)

Brady, S., Bogossian, F., y Gibbons, K., The effectiveness of varied levels of simulation fidelity on integrated performance of technical skills in midwifery students - A randomised intervention trial, https://doi.org/10.1016/j.nedt.2014.11.005, Nurse Education Today, 35(3), 524-529 (2015)

Cheng, A., Lockey, A., y otros cuatro autores, The use of high-fidelity manikins for advanced life support training--A systematic review and meta-analysis, https://doi.org/10.1016/j.resuscitation.2015.04.004, Resuscitation, 93, 142-9 (2015) 
Corvetto, M., Bravo, M.P., y otros seis autores, Simulación en educación médica: una sinopsis, http://doi.org/10.4067/S0034-98872013000100010, Rev. Méd. Chile, 141(1), 70-79 (2013)

Coyer, C., Gascoin, G., y otros cuatro autores, Evaluation of the impact and efficiency of high-fidelity simulation for neonatal resuscitation in midwifery education, https://doi.org/10.1016/j.arcped.2014.06.010, Arch. Pediatr., 21(9), 968$975(2014)$

Dearmon, V., Graves, R.J., y otros seis autores, Effectiveness of simulation-based orientation of baccalaureate nursing students preparing for their first clinical experience, https://doi.org/10.3928/01484834-20121212-02, The Journal of Nursing Education, 52(1), 29-38 (2013)

Haro, M., Autoconfianza versus autoeficacia del traductor: propuesta terminológica y estado de la cuestión, https://doi.org/10.5007/2175-7968.2019v39n2p204, Cad. Trad., 39(2), 204-226 (2019)

Hicks, F.D., Coke, L., y Suling, L., The Effect of High-Fidelity Simulation on Nursing Students' Knowledge and Performance: A Pilot Study, NCSBN Research Brief, 40, Chicago, USA (2009)

House, S., Dowell, S., y otros tres autores, Low-fidelity simulation to enforce patient safety, https://doi.org/10.1016/j.ecns.2015.11.002, Clinical Simulation in Nursing, 12(1), 24-29 (2016)

Huang, J., Tang, Y., y otros ocho autores, Educational efficacy of high-fidelity simulation in neonatal resuscitation training: a systematic review and meta-analysis, https://doi.org/10.1186/s12909-019-1763-z, BMC Medical Education, 19, 323 (2019)

Juguera, R.L., Díaz, J.L., y otros cuatro autores, La simulación clínica como herramienta pedagógica: percepción de los alumnos de Grado en Enfermería en la UCAM (Universidad Católica San Antonio de Murcia), Enfermería Global, ISSN: 1695-6141, 13(33), 175-190 (2014)

Kawarai, A., Harada, K., Kawahira, H., y Mitsuishi, M., The effect of simulator fidelity on procedure skill training: a literature review, https://DOI: 10.5116/ijme.5ea6.ae73, International Journal of Medical Education, 11, 97-106 (2020)

Kennedy, C.C., Cannon, E.K, Warner, D.O., y Cook, D.A., Advanced airway management simulation training in medical education: a systematic review and meta-analysis, https://doi.org/10.1097/ccm.0b013e31829a721f, Critical Care Medicine, 42(1), 169-178 (2014)

Kononowicz A.A., Woodham L.A., y otros ocho autores, Virtual Patient Simulations in Health Professions Education: Systematic Review and Meta-Analysis by the Digital Health Education Collaboration, https://doi.org/10.2196/14676, J. Med. Internet Res., 21(7): e14676 (2019)

Laschinger, S., Medves, J., y otros cinco autores, Effectiveness of simulation on health profession students' knowledge, skills, confidence and satisfaction, https://doi.org/10.1111/j.1744-1609.2008.00108.x, International Journal EvidenceBased Healthcare, 6(3), 278-302 (2008)

Martínez, G.A., y Jiménez, N., Análisis del uso de las aulas virtuales en la Universidad de Cundinamarca, Colombia, http://doi.org/10.4067/S0718-50062020000400081, Formación Universitaria, 13(4), 81-92 (2020)

Martínez, C.F., y Matus, M.R., Desarrollo de habilidades con simulación clínica de alta fidelidad, perspectiva de los estudiantes de enfermería, https://doi.org/10.1016/J.reu.2015.04.003, Enfermería Universitaria, 12(2), 93-98 (2015)

Mould, J., White, H., y Gallagher, R., Evaluation of a critical care simulation series for undergraduate nursing students, https://doi.org/10.5172/conu.2011.38.1-2.180, Contemporary Nurse, 38(1-2), 180-190 (2011)

Mundell, W., Kennedy, C., Szostek, J., y Cook, D., Simulation technology for resuscitation training: a systematic review and meta-analysis, https://doi.org/10.1016/j.resuscitation.2013.04.016, Resuscitation, 84(9), 1174-1183 (2013)

Náñez, J.J., Ramírez, A.E., y González, A.M., Factores asociados al componente pedagógico del uso ocupacional del laboratorio de simulación de las facultades de ciencias de la salud en Colombia, Revista Ideales, ISSN: 2539-5211, 9(1), 45-58 (2019)

Rubio, G., Putet, G., y otros once autores, In situ simulation training for neonatal resuscitation: an RCT, https://doi.org/10.1542/peds.2013-3988, Pediatrics, 134(3), e790-e797 (2014)

Serna, D.C., y Martínez, L.S., La simulación en la educación médica, una alternativa para facilitar el aprendizaje, https://doi.org/10.30554/archmed.18.2.2624.2018, Archivos de Medicina (Col), 18(2), 447-454 (2018)

Verkuyl, M., Lapum, J., y otros cuatro autores, Exploring debriefing combinations after a virtual simulation, https://doi.org/10.1016/j.ecns.2019.12.002, Clinical Simulation in Nursing, 40, $36-42$ (2020)

Weller, J.M., Nestel, D., y otros tres autores, Simulation in clinical teaching and learning, https://doi.org/10.5694/mja10.11474, Medical Education, 196(9) (2012)

Zhang, H., Wang, W., y otros tres autores, The impact of a three-phase video-assisted debriefing on nursing students' debriefing experiences, perceived stress and facilitators' practices: A mixed methods study,

https://doi.org/10.1016/j.nedt.2020.104460, Nurse Education Today, 90, 1-8 (2020) 